\title{
The promise of metamaterials for new applications in optics
}

H. Schweizer, L. Fu, N. Liu, T. Weiss, P. Schau, et al.

H. Schweizer, L. Fu, N. Liu, T. Weiss, P. Schau, K. Frenner, W. Osten, H. Giessen, "The promise of metamaterials for new applications in optics," Proc. SPIE 8083, Modeling Aspects in Optical Metrology III, 808302 (8 July 2011); doi: $10.1117 / 12.895026$

SPIE. Event: SPIE Optical Metrology, 2011, Munich, Germany 


\title{
The Promise of Metamaterials for New Applications in Optics
}

\author{
H. Schweizer ${ }^{* a}$, L. Fu ${ }^{a}$, N. Liuc, T. Weiss ${ }^{a}$, P. Schau ${ }^{b}$, K. Frenner ${ }^{b}$, W. Osten ${ }^{b}$, \\ and H.Giessen ${ }^{a}$ \\ ${ }^{\text {a }} 4^{\text {th }}$ Physics Institute, Universität Stuttgart, Pfaffenwaldring 57, 70550 Stuttgart, Germany \\ ${ }^{b}$ Institute für Technische Optik, Universität Stuttgart, Pfaffenwaldring 9, 70569 Stuttgart, \\ Germany \\ ' Department of Chemistry, University of California, Berkeley, and Materials Sciences Division, \\ Lawrence Berkeley National Laboratory, Berkeley, California 94720
}

\begin{abstract}
Metallo-dielectric structured materials, or in other words metamaterials (MTM), are in principle a well established composite to improve efficiency, functionality, and weight of micro-wave components. In recent times, it has been demonstrated that the functionalities of metamaterials can be scaled down to optical frequencies by nano structuring techniques. Examples include negative index materials in the near infrared and visible frequency range, cloaking structures, filters, and structures for improved sensing of environmental gases.

The physical processes in plasmonic metamaterials depend strongly on the excitation of surface plasmons and the interaction between them.

We have learned how to control the plasmon-photon and the plasmon-plasmon interaction for manipulating the electromagnetic response in a metamaterial at wavelengths well below the vacuum wavelength. Many interesting and novel optical applications and devices are expected. For instance sub-wavelength imaging, compact communication devices as polarisation splitters, slow light media structures, compact colour filters, and resonators. All-plasmonic circuits are also the basis for ultra-dense photonic integration not achievable through the conventional optical integration.

With examples of several metamaterial structures we try to illustrate the application potential of MTMs and comment on their fabrication feasibility to show whether metamaterials can hold their promise. Their investigation is in any case a rewarding adventure.
\end{abstract}

\section{History of Metamaterials}

Metamaterials (MTM) are manmade metallo-dielectric composite structures with structure sizes below the operation wavelength of the electromagnetic vacuum wave used. The metallo-dielectric structures allow control over the electromagnetic material parameters such as permittivity $(\varepsilon)$ and permeability $(\mu)$ or the product of both, the refractive index $\mathrm{n}$. The history of the recent 70 years showed that metallo-dielectric structures have already been used in the field of microwaves and radio frequencies to reduce the weight and functionality of lens devices [1]. In those applications the MTM can be represented by waveguide like media [1] or by small metallic inclusions (metallic spheres) in the dielectric host matrix [2], replacing heavy, high-permittivity materials by much lighter composites comparable permittivity. These early attempts showed that the engineering principle applied for metamaterial designs is:

1) design a new material by changing the effective refractive index with the help of metallic small composites

2) give the new material a suitable functional shape which may deviate from the standard shapes of the devices made by conventional materials

In some cases (see microwave applications [3] of MTMs) the shape of functional devices in the case of waveguides, couplers, and antennas can be changed to more suitable geometries. Already in this early stage one observed a characteristic property of MTM structures: their scaling behaviour. MTM structures can be scaled down from low to high frequencies by simply changing their geometry but maintaining the principal geometrical shape of the inclusion unit cell. This can be nicely illustrated by an example of a meander line structure which is used as phase retarder. The meander line structure works as an inductive (phase advance) or a capacitive (phase delay) element according to the polarization of the incident electromagnetic wave. Therefore, it can work as a phase retarder at very different frequencies, i.e., in the low $\mathrm{GHz}$ range [4], in the E-band range (69-90 GHz) [5] and even in the IR-frequency range (25-37 THz) [6]. Thereby MTM-structures achieve a high efficiency with only a few functional layers. Most importantly, in some frequency ranges there is no alternative natural material to achieve such an effect. However, Modeling Aspects in Optical Metrology III, edited by Bernd Bodermann, Harald Bosse, Richard M. Silver, Proc. of SPIE Vol. 8083, 808302 · @ 2011 SPIE · CCC code: 0277-786X/11/\$18 · doi: 10.1117/12.895026 
principal scaling limits are also noticed. They are induced by the kinetic energy of electrons contributing to the inductance of a structures which prevents a down scaling to arbitrary small structures [7].

In recent times new interest on MTM structures was coming up due to the publications of Veselago [8] and Pendry [9] who pointed out that materials with negative refractive index can play an important technological and scientific role if they can be realized at technological interesting wavelengths. Pendry argued that slabs of materials with negative refractive index (negative index $\boldsymbol{m}$ aterial (NIM)) not only show an imaging behaviour for travelling waves as already noticed by Veselago [8] but also for evanescent waves. Combinations of positive index materials (positive index $m$ aterial (PIM)) and NIM forming complementary media [10] can cancel the optical space between two real space points, and can therefore work as a perfect lens. In such a lens, not only travelling waves but also evanescent waves are reproduced in the object image, thereby achieving a sub-wavelength resolution (see perfect lensing theorem (PLT) [10]). This perfect lensing concept was exciting enough to start the race for hunting the best MTM structures for NIM.

\section{The role of plasmons}

In the analysis of the perfect lens it becomes aware that surface waves (surface plasmons (SP) [11]) play a decisive role in the physical mechanism. The surface plasmons are even indispensable in coupling the evanescent field to the 'lens device' since only SPs, due to their dispersion relation [11], have high enough momentum to couple with the evanescent fields. An analysis of the boundary conditions $[12,13]$ for plane waves in a NIM slab reveals that the bulk NIM can be replaced by two thin surface layers. They act as phase conjugating layers $[12,14]$ or as resonantly coupled double layers [13] which are just a pair of frequency selective surfaces (FSS). This is a basis, as we will see below, to overcome inevitably present losses (due to dispersion [15]) in pure NIM structures.

\section{Promises}

The coupling mechanism of plasmons in MTM-NIM structures not only opens the field for new optical applications in the direct sense but also to wider fields. The coupling property between plasmons and evanescent fields is also the basis to couple other sub-wavelength sources such as quantum dots and dot like emitters such as nano diamonds [16] very efficiently to plasmon waveguide media, opening the field of quantum communication. A review article of Barnes [17] showed that the integration potential of plasmons can overcome the integration density of modern SOIbased (Silicon-on-Insulator [18]) by a factor 50-100 if we compare the SP wavelengths and decay lengths in the metal plasmon waveguide with the photon wavelength in vacuum or in the surrounding dielectrics. The review article of Ozbay [19] shows also the potential of all-plasmonic circuits with respect to couplers, and waveguide miniaturization. The plasmonic circuits can overtake the function of photonic circuits at much smaller length scales comparable with electronic integrated circuits. This is shown by the group of Bozhevolnyi [20] demonstrating Y-couplers, MachZehnder interferometers and ring resonator structures in the micrometer-ranges which show a smaller length scale than the standard photonic integrated circuits [18].

The application of MTM-NIM structures in the field of photonics relies strongly on their homogeneity. When this problem is solved fascinating, devices on the basis of PLT [10] as super scatterers [21] and super phase arrays [22] can be achieved. In both applications the PLT is applied to curved spaces by transformation optics [10, 23-27] thereby introducing optical magnification which is not possible in the simple NIM slab waveguide. The optical magnification can increase the virtual dimension of an electromagnetic object appreciably compared to its physical size [10]. Interesting applications to antennas show up, where the directivity in relation to beam steering of an antenna was optimized with a NIM-lens [22] even in the presence of losses [21]. Also the object (antenna or scatterers) can be realized in more compact way. An antenna on this basis would be also more energy efficient.

The scaling behaviour of MTM-structures also results in the hope that MTM-NIM applications achieved in the field of microwaves [3, 28], e.g. compact directional couplers, compact antennas, dual band devices, impedance matched lenses and compact resonators (based on complementary MTM material structures) can be transferred to the optical regime and also vice versa. The ladder is normally easy to realize, but the former experiences more problems since MTM structures with discrete unit cells are not always in the range $P / \lambda \ll 1$ (with $\boldsymbol{P}$ the structure size $\lambda$ the operation wavelength). This results besides the local response of the MTM unit cells in non-local interactions. Weak and strong spatial dispersion can occur by the hybridization of plasmon modes [29,30] or by chiral effects [31]. Spatial dispersion effects can be even present in the limit of large values of $\lambda$ as the case of uniaxial wire media [32] demonstrates. A closer inspection of these effects is therefore necessary to engineer MTM-NIMs. 


\section{Nanostructured MTM: Plasmon-Plasmon coupling / the impact of spatial dispersion}

Recently different authors [33 - 36] have analyzed the possibility of realizing nanostructured MTMs which can be described in a certain $\boldsymbol{P} / \boldsymbol{\lambda}$ range [35] as MTM-NIM which means a characterization by two effective parameters: permittivity $(\varepsilon)$ and permeability $(\mu)($ Eq. 1a, b)

$$
\begin{aligned}
& \vec{D}(\vec{r}, \omega)=\varepsilon_{0} \cdot \bar{\varepsilon}(\omega) \cdot \vec{E}(\vec{r}, \omega) \\
& \vec{B}(\vec{r}, \omega)=\mu_{0} \cdot \bar{\mu}(\omega) \cdot H(\vec{r}, \omega)
\end{aligned}
$$

(with artificial permittivity $\varepsilon(\omega)(\operatorname{Re}[\varepsilon(\omega)]<0)$ and artificial permeability $\mu(\omega)(\operatorname{Re}[\mu(\omega)<0]))$

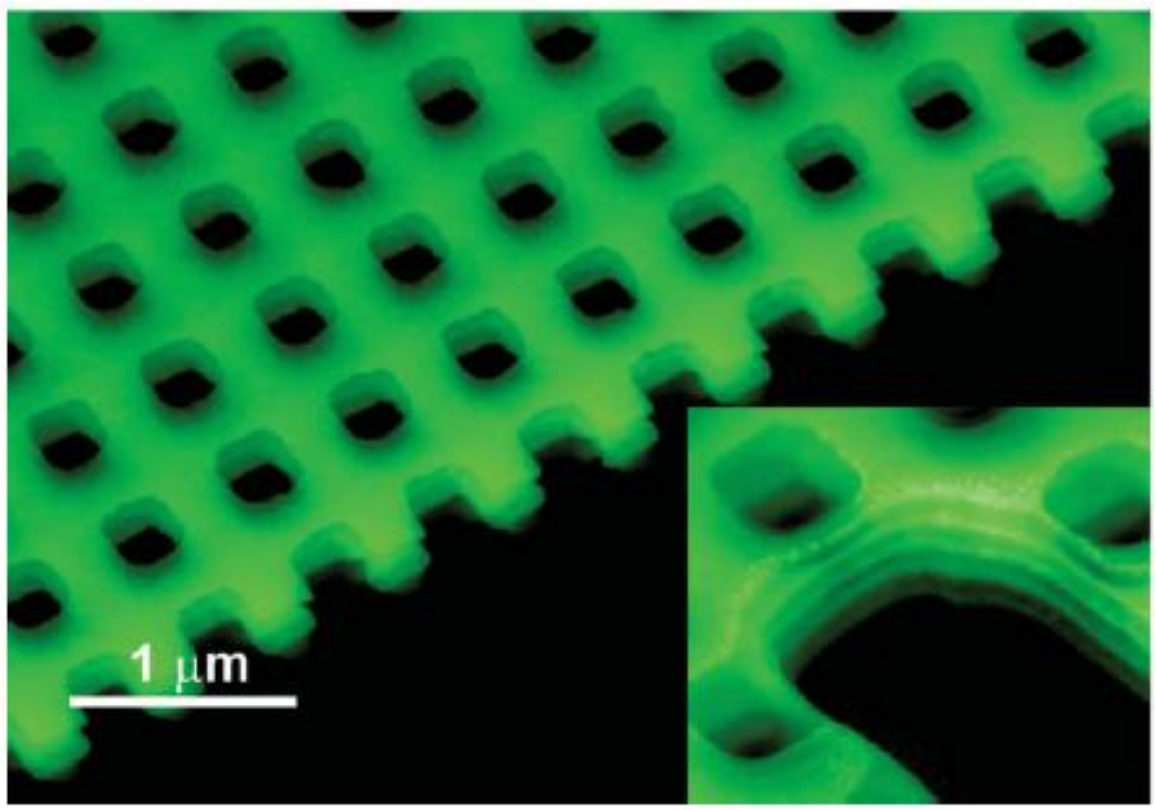

Fig. 1: SEM image of fabricated 5-layer fishnet metamaterial. The inset shows a focused-ion-beam cut of the sample demonstrating the layer number of the fabricated structure [30].

The MTM might be understood as a continuous material as discussed in the sense above with all benefits of a continuous NIM, e.g. to realize perfect lenses and compact optical devices etc. The permeability can appear as artificial magnetics due to non-local spatial dispersion effects of $2^{\text {nd }}$ order [35] as already mentioned in the past [15]

$$
\mu_{k l}=\left[\delta_{k l}-\mu_{0} \omega^{2} \gamma_{k l}\right]^{-1}
$$

(with $\gamma_{\mathrm{kl}}=2^{\text {nd }}$ order dispersion coefficient related to $\xi_{\mathrm{kmnl}}[36,39]$ ).

The result found in [35] is that a NIM (in the analyzed case a fishnet structure [37]) shows a homogeneous NIM behaviour in a very tight $\mathrm{P} / \lambda$ - range $(0.15-0.25)$. However for most of the interesting ranges of $\operatorname{Re}(\mu)<0$ the response is based on non-local weak dispersion effects (WSD) which force the introduction of additional parameters $\psi, \xi, \chi[33]$ in the constitutive material equations [34] (Eq. 3a,b) in addition to $\varepsilon$ and $\mu$.

$$
\begin{aligned}
& D_{k}(\vec{r}, \omega)=\left(\varepsilon_{0} \cdot \varepsilon_{k l}\right) \cdot E_{l}-\Psi_{l k} H_{l}+\xi_{k m n l} \frac{\partial^{2} E_{l}}{\partial x_{m} \partial x_{n}} \\
& B_{k}(\vec{r}, \omega)=\left(\mu_{0} \cdot \mu_{k l}\right) \cdot H_{l}+\Psi_{k l} E_{l}+\chi_{k m l} \frac{\partial E_{l}}{\partial x_{m}}
\end{aligned}
$$

(with permittivity $\varepsilon_{\mathrm{kl}}$; bianisotropy coefficient $\Psi_{\mathrm{k} ।}=-\Psi_{\mathrm{lk}}$; magnetoelectric coefficient $\chi_{\mathrm{klm}}$; and quadruple coefficient $\xi_{\mathrm{kmnl}}$ ) 
Equations $3 \mathrm{a}, \mathrm{b}$ are a consequence of the fact that in reciprocal media (time-even-media) the polarization is due to electric polarization (time-even-process, chap. 7 in [38]) whereas magnetic fields (time-odd-quantity) do not induce directly polarization as they cannot do work classically [39,40]. This results in a relation between currents (induced or external) and electric fields. In the simplified form by a Taylor series (Eq. 3a, b) introducing $1^{\text {st }}-$ and $2^{\text {nd }}$-order spatial dispersion coefficients $\psi, \xi, \chi$ besides $\varepsilon$ and $\mu$ by the respective spatial derivatives of appropriate order of the electric field. Indeed, in realized fishnet structures [30] (Fig. 1) we observe a layer number dependent permeability and permittivity if interpreted in the usual way [41-43] (Fig. 2).

These observations deserve as already mentioned in [30] special care in interpretation of numerical results with respect to NIM effects [33,34]. Furthermore under systematic variation of the layer number one observes strong interaction between layers which could be nicely explained by a plasmon hybridization effect [44] of the magnetic plasmon modes.

\section{Examples of coupled nano systems}

Plasmonic coupling can be directly investigated in stacked nanometer structures [29] or between lateral clusters of plasmonic particles [50]

The systematic variation of layer distances in 3D-U-shaped metamaterial stacks [30] shows by increasing layer distances a decrease of the symmetric plasmon mode in frequencies and an increase of the antisymmetric plasmon in frequencies. The type of modes was identified from numerical calculations of the current distribution in the U-stacks. The case of stacked nanostructured MTM is most interesting for identification of plasmon-plasmon interactions. With respect to a unique parameter attribution the situation is actually open.

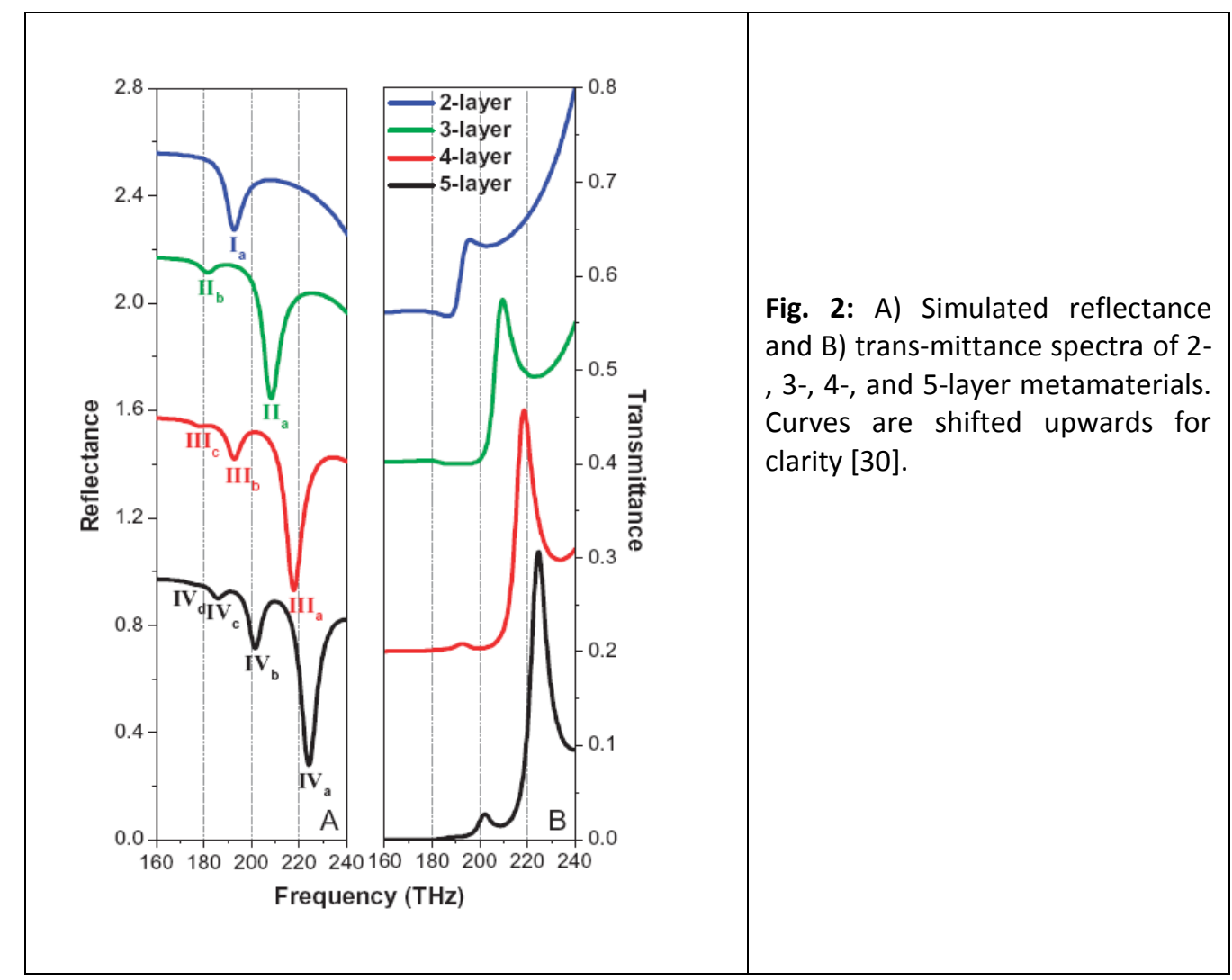

It is clear that in most cases of MTM structures the attribution of only two parameters $\varepsilon$ and $\mu$ is not possible [34] and further parameters describing magnetoelectric coupling (bi-isotropic/anisotropic, chiral effects) by $1^{\text {st }}$ order weak spatial dispersion (WSD) [35] and artificial permeability by $2^{\text {nd }}$ order WSD effect must be taken into account. In addition higher multipole excitations in the unit cell as a further variant of a WSD -effect [40] can also be observed in the case of metamaterials of twisted stacked U-shaped particles [45]. From the discussion in [34] it becomes obvious that any non-uniform current distribution in more or less complicated metallo-dielectric inclusion geometries can result in $1^{\text {st }}$ and $2^{\text {nd }}$ order spatial dispersion effects. Where $1^{\text {st }}$ order WSD effects can result in bi-(an)isotropy or 
chirality and $2^{\text {nd }}$ order WSD effects can result in artificial magnetic effects (see Eq. 2). The occurrence of artificial magnetization is due to the fact that the polarization fields (Eq. 4a, b) in

$$
\begin{aligned}
& \vec{D}^{\prime}=\vec{D}+\nabla \times \vec{Q} \\
& \vec{H}^{\prime}=\vec{H}+\partial Q / \partial t
\end{aligned}
$$

an electromagnetic body are not uniquely defined [39] but are undefined up to an arbitrary differentiable vector function Q (Eq. 4a, 4b) [35, 39, 46] which can be suitably chosen to obtain transformed fields

which obey the standard boundary conditions known from homogeneous materials: continuous tangential field components of $E$ and $H$ and continuous normal field components of $D$ and $B$. A choice of $Q=j \omega \gamma B$ (for $e^{+j \omega t}$ dependence) according to Eq. 5 gives a new set of equations

$$
\vec{Q}=-\gamma \cdot \nabla \times \vec{E} \equiv j \cdot \omega \cdot \gamma \cdot \vec{B}
$$

of the induced polarization where the curl-term in the electric polarization [39] can be transformed and a new term of $2^{\text {nd }}$ order spatial dispersion appears in the magnetic polarization $\mu$ (Eq. 2, 6c). Eqs. 6a-6c are simplified expressions valid for the biisotropic case [39].

$$
\begin{aligned}
& \vec{D}^{\prime}=\varepsilon \cdot \vec{E}-j \cdot \xi \cdot \vec{B}+\beta \cdot \nabla \nabla \vec{E} \\
& \vec{H}^{\prime}=\mu^{-1} \cdot \vec{B}-j \xi \cdot \vec{E} \quad \text { and } \\
& \mu=\mu_{0} /\left(1-\omega^{2} \cdot \mu_{0} \cdot \gamma\right)
\end{aligned}
$$

(with $\xi, \beta, \gamma=1^{\text {st }}-, 2^{\text {nd }}$-order spatial dispersion coefficients and standard $\varepsilon, \mu$ parameter)

The artificial magnetic effect (Eq. 6c) which is a $2^{\text {nd }}$ order WSD effect is dominated by $1^{\text {st }}$ order WSD effects if $1^{\text {st }}$ order effects in the Taylor series (Eq. 3a, 3b) are not suppressed by symmetry. If a pure artificial magnetic effect is desired the bi-(an)isotropy must be suppressed by structures which contain inversion symmetries or mirror symmetries of the form $\sigma_{x}, \sigma_{y}, \sigma_{z}$. This works well at $\mu$-wave frequencies [47] but might be critical at visible frequencies. A suitable proposal using nano rings of plasmonic nano spheres is given in [48] to realize an effective permeability without bianisotropy. This is also possible with the concept of core shell particles using Mie scattering [49]. A detailed study on resonant coupling of plasmonic spheres experimentally and theoretically for optical frequencies is given by Hentschel [50]. This study exceeds studies on dimers [44] in the past and investigates in detail hexamer and heptamer particle clusters. As function of inter particle distance $d(20 \mathrm{~nm}-130 \mathrm{~nm})$ the authors could demonstrate transition from an isolated single plasmon particle mode to collective modes resembling the process of molecule orbital formation. Even a seemingly small change in particle geometry from a hexamer to a heptamer results in a drastic change of the optical transmittance spectra indicating that the description of MTM structures with rather complicated metallodielectric geometries with only a few optical parameters as permittivity, permeability, chiral parameter and quadrupole parameter turns out to need more research.

This can be further seen when already rather simple MTM structures such as metallic films with holes are investigated. In a recent publication Gompf and co-workers [31] measured strong polarization rotation in a squared hole array of an Au film at oblique incidence. The investigated structure was measured in a $P / \lambda$ parameter range $(P=$ structure period, $\lambda=$ operation wavelength) between $1.5-0.23$. For these $P / \lambda$ - parameter values spatial dispersion effects [35] should show up resulting in a description of the structure with more than two parameters.

According to $[33,34]$ besides permittivity $(\varepsilon)$ and permeability $(\mu)$ a bi-anisotropic and quadrupole contribution (see Eq. 3a, 3b) can occur in the constitutive equations. And the bi-anisotropic contribution can result in a chiral contribution ( $\Psi$ symmetric) or in an $\Omega$-medium contribution ( $\Psi$ anti-symmetric). The squared hole array analyzed in [31] should have mainly $k^{2}$-dependend contributions since the $k$-linear contributions vanish in this structure with inversion symmetry [39] if not quadrupolar WSD-effects $[34,40]$ must be taken into account. In [31] the observed rotation power (ODR = optical dispersion rotation) is appreciably larger than an optically active material like quartz of same layer thickness. One obtains for the MTM structure compared at the same layer thickness a 5 orders of magnitude higher value than with the natural material quartz. 
The difference between MTM structure and natural material might be explained by strong resonant plasmonic contributions [34], which are also noticed in other experiments on plasmon-induced magneto optical activity [51].

\section{Frequency Selective Surfaces (FSS)}

The plasmon enhancement of the optical response can be observed in an important class of MTM materials: resonantly coupled frequency selective surfaces already mentioned above $[13,14]$ show strong resonant plasmon based transmission effects. If the FSS structure consists of metallic ( $\mathrm{Au}$ ) meander film geometries the optical response of the coupled films can be designed to show bi-anisotropy as $\Omega$-medium or to show artificial magnetism by plasmon enhancement 2 nd order WSD effects.

Coupled FSS structures can mimic NIM material slabs $[10,12,13]$ promissing to reduce losses in the MTM-NIM. A coupled FSS structure can be realized by a double meander structure. The double meander structures show $\mathrm{C}_{2}$ symmetry and inversion symmetry [52]. Therefore $1^{\text {st }}$ order dispersion effects should be small and $2^{\text {nd }}$ order WSD effects should be present. Due to resonant plasmon coupling we observe a tremendous increase in transmittance. This enhancement of the $2^{\text {nd }}$ order effect is due to the resonant plasmon effect which can be observed already in single meander layers. Thin metal films show electromagnetic surface waves (plasmons) [11]. According to the symmetry of the excited charge distribution, long range surface plasmon polaritons (LRSPP) or short range plasmon polaritons (SRSPP) with asymmetric and symmetric charge distribution can be attributed, respectively. When a metal film is corrugated (meander surface) (Fig. 3 left) the dispersion relation

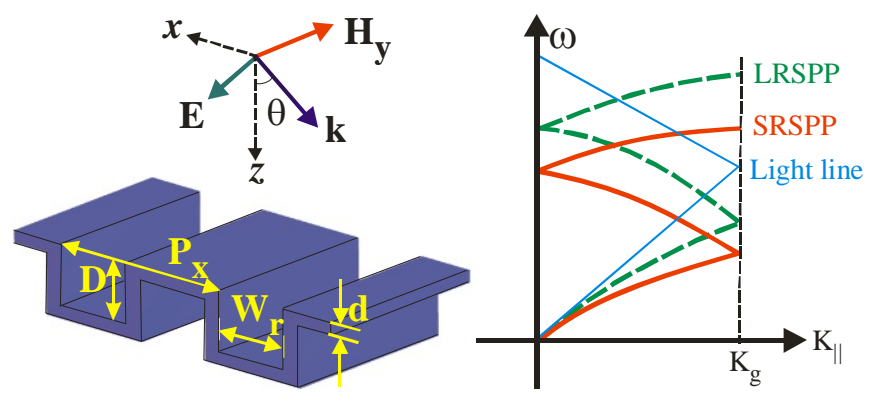

Fig. 3: Left: Principal of a single meander meta surface (MMS) structure. $\mathrm{P}_{\mathrm{x}}=$ corrugation period; $\mathrm{D}=$ corrugation depth; $\mathrm{W}_{\mathrm{r}}$ $=$ meander width; $\mathrm{d}=$ metal thickness.

Right: Sketch of a dispersion relation of photons and LR/SRSPP

plasmons of a MMS structure charac-terized by the half reciprocal lattice vector $\mathrm{K}_{\mathrm{g}}=\pi / \mathrm{P}_{\mathrm{x}}$.

of the SR/LRSPP modes [53-55] can be folded into the light cone (Fig. 3, right) thus fulfilling the momentum conservation between plasmon and photons. LRSPP- and SRSP- modes of the top and bottom side of the meander film couple resonantly showing enhanced transmission [54] (Fig. 4). Compared to the closed uncorrugated film the enhancement of the transmission exceeds the value of the 

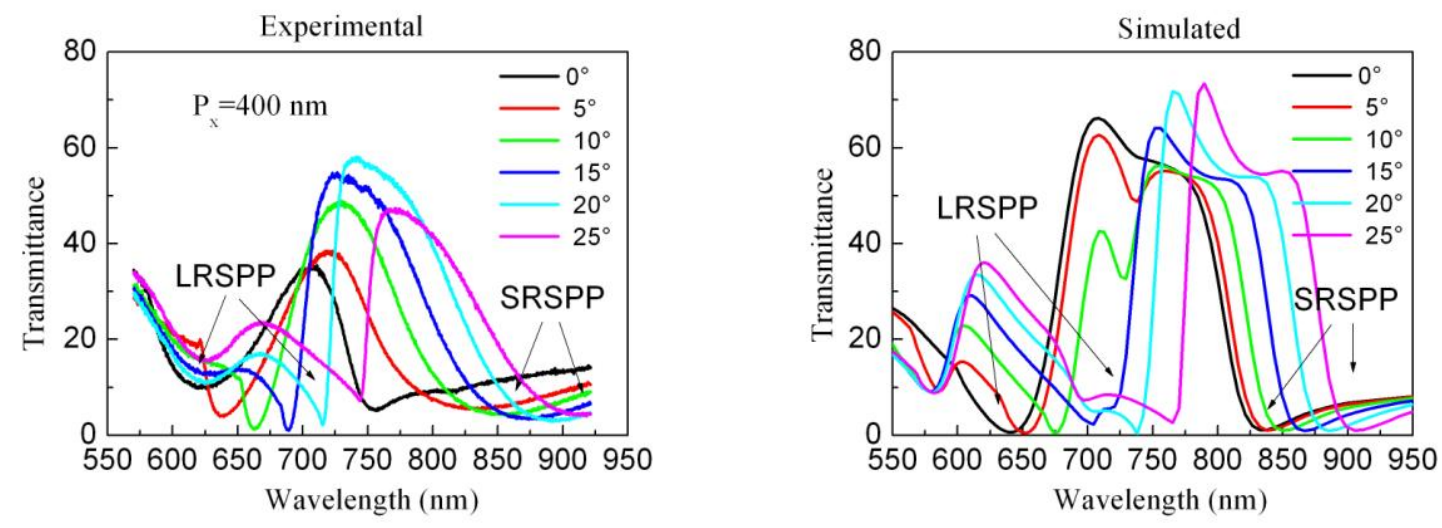

Fig. 4: Transmission spectra (p-polarization) of an Au-MMS structure on $\mathrm{SiO} 2(\mathrm{nSiO} 2=1.46)$ dielectric layer with structure parameters (parameter definition see Fig. 3) Px $=400 \mathrm{~nm}, \mathrm{D}=40 \mathrm{~nm}, \mathrm{Wr}=\mathrm{Px} / 2-\mathrm{d}, \mathrm{d}=30 \mathrm{~nm}$ (Au). Left: Experimental transmission spectra. Right: Simulated transmission spectra (for Au we used Johnson-Christy dispersion data [56]). The angles of incidence are coded by differently coloured traces $\left(0^{\circ}-25^{\circ}\right)[54]$.

smooth film by more than a factor of 20 . We named the corrugated meander metal films as meander meta surfaces (MMS) [54] The resonant coupling of LR / SR plasmons form a resonant passband $[57,58]$ which shows negative dispersion $[53,54]$ with respect to the transversal momentum.

A transmission line analysis $[53,59,60]$ reveals that the SRSPP modes belong to magnetic dipole excitation in the sense of artificial magnetics $[33,34]$ and the LRSPP to an electric dipole excitation. We analyzed the single meander structure also with a Fourier modal method [61] (or sometimes called rigorous coupled wave analysis (RCWA) [62]) for solving the Maxwell equations. In [62] we modified the RCWA on the basis of normal vector fields (NV-method [63]) by adopting the method of Fast Fourier Factorization [64] to find an optimized solution for application of Li's [65] factorization rules. In [61] we modified the RCWA also by LI's factorization rules and application of adaptive spatial resolution (ASR) $[66,67]$. From the scattering matrix algorithm we calculated transmission and reflexion for all diffraction orders taking into account a sufficient number (for the MMS structures 61 plane waves) of harmonic waves to obtain a good convergence. In Fig. 4 a comparison between experimental and simulated single meander transmission spectra are depicted for different angles of incidence $\left(0^{\circ}-25^{\circ}\right)$. The agreement between theory and experiment is quite satisfactorily if one keeps in mind that we took realistic material constants for gold [56] and used no further fitting in the calculation. According to the dispersion relation of the surface plasmon polaritons (SPP) [53, $55,57]$ the passband shifts for increasing incident angle to the red. Also the SR/LRSPP stop bands indicates negative dispersion with respect to the transversal momentum $\left(k_{t}=(\omega / c) \sin \theta, \theta=\right.$ incident angle). As mentioned by Maslovski $[12,13]$ and Wee, Pendry [14] a negative index material may be mimicked [68] by a combination of phase conjugation surfaces $[12,14]$ in conjunction with low loss positive index materials or resonantly coupled frequency

(a) Extinction

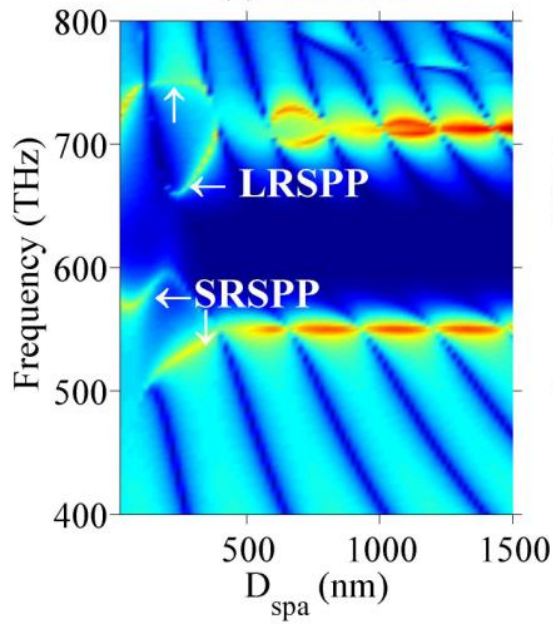

(b) Transmittance

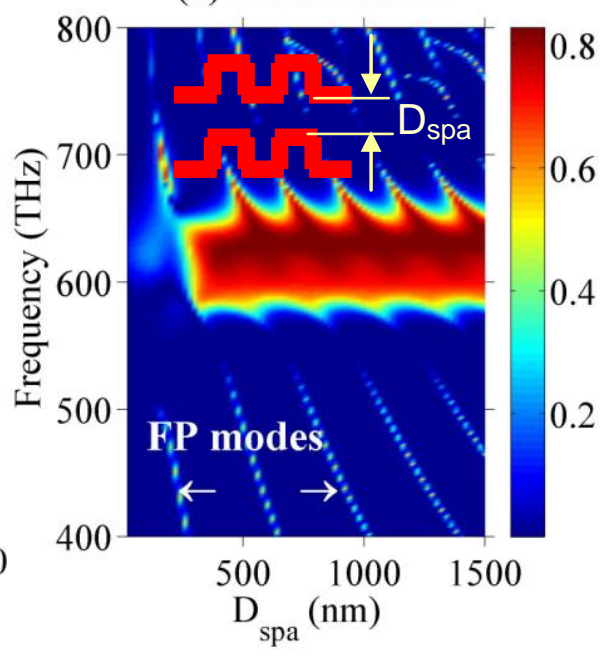

Fig. 5:

Extinction (left) and transmittance (right) spectrum of a double Ag-MMS structure as function of MMS distance $\mathrm{D}_{\mathrm{spa}}$. [54]. The struc-tural parameters of each meander layer are as in Fig. 3. Material parameters see $[69$, 70] 
selective surfaces [13]. As an advantage of coupled FSS structures over bulk NIM-MTM the possibility appears that optical losses are appreciably reduced since most of the structure volume consists of low loss transparent material. Before we discuss applications of the resonantly coupled meta surfaces we want to discuss more in detail the properties of coupled meta surfaces when the distance $D_{\text {spa }}$ between layers is varied. The understanding of this property is very important for engineering more complicated device structures to realize magnification [10] or even more complicated filter functions [23, 24]. The study of resonantly coupled MMS structures ends in a surprise if the distance between layers is varied (Fig. 5a, b). As seen in Fig. 5b the coupled

MMS structure not only behaves as a Fabry-Perot cavity but also in a frequency range of plasmon resonances (SR/LRSPP) the resonant transmission can be kept over a rather large distance. This effect shows that on the basis of plasmon polaritons large distances in a material can be bridged nearly lossless, an indispensable property for engineering MTM-devices with larger volumes [71]. This is also the basis to obtain superlenses

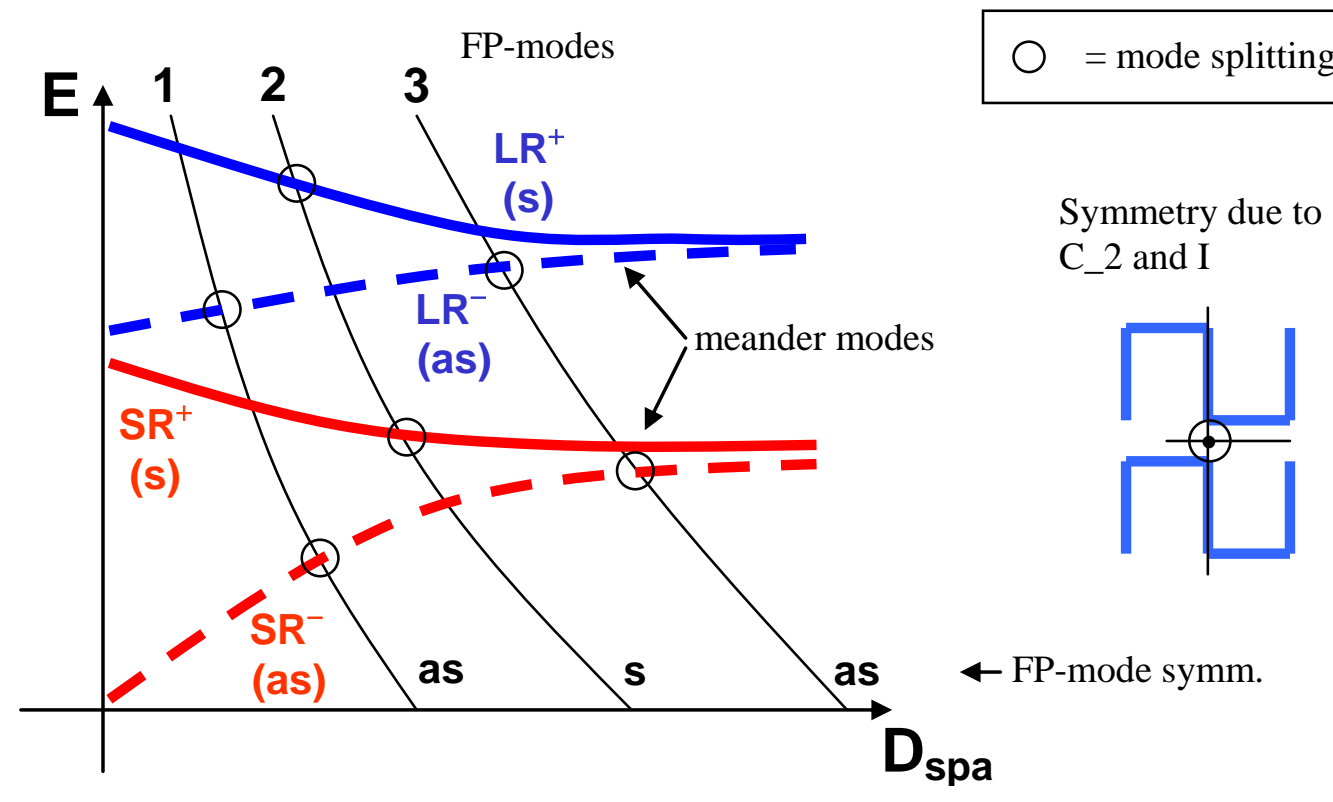

Fig. 6: Principal mode dispersion of plasmon polariton- and FP-modes in a resonantly coupled double MMS structure. LRSPP and SRSPP modes show for smaller distances $D_{\text {spa }}$ mode splitting in s- $\left(\mathrm{SR}^{+}, \mathrm{LR}^{+}\right)$and as- $\left(\mathrm{SR}^{-}\right.$, $\mathrm{LR}^{-}$) modes with respect to $\mathrm{C}_{-} 2$. The FP-mode series starts with respect to $\mathrm{C} 2$ with an antisymmetric mode followed by symmetric modes and so on. Mode splitting (open circles) is observed between SPP- and FPmodes of same symmetry [52].

[14, 72-75]. A detailed study [52] of the interaction between FP-modes (hyperbolic curves in Fig. 5a, b) shows a complex mode interaction seen in more detail in the extinction spectrum (Fig. 5a). A closer inspection [52] of the symmetries of plasmon- and FP-modes reveals that SPP- and FP- modes of same inversion- or $\mathrm{C}_{2}$ - symmetry show strong mode splitting (Fig. 6, and Fig. 5a). In addition, with decreasing meander distance $D_{\text {spa }}$ the SPP modes, both SRand LRSPP modes show increasing splitting in symmetric / antisymmetric modes (Fig. 5a, and Fig. 6) according symmetry operation $C_{2}$ thereby shifting the $C_{2}$ even modes $\left(\mathrm{LR}^{+}, \mathrm{SR}^{+}\right)$to higher frequencies and the $\mathrm{C}_{2}$-odd modes $\left(L R^{-}, S R^{-}\right)$to lower frequencies. Since the first FP-mode is $C_{2}$-odd one observes a strong mode splitting between the $\mathrm{FP}$ - and the antisymmetric $\mathrm{LR}^{-}$- and $\mathrm{SR}^{-}$- plasmon modes suppressing the resonant transmission through the double surfaces for the first $250 \mathrm{~nm}$ (Fig. 5b) in the given case. The effect appears to be rather stable since for the $\mathrm{Ag}$ material parameters experimentally given parameters $[69,70]$ are introduced. 


\section{Applications of FSS}

MTM structures which can mimic negative index behaviour can be used for near field imaging [8, 9] and further applications of the perfect lens theorem (PLT) [10] as super scatterers and super phase arrays [14, 21-27]. As mentioned above the MMS-structure is indispensable for coupling evanescent fields with plasmon fields to realize practical devices based on PLT. This can be demonstrated with a resonantly coupled double MMS structure

(Fig. 7). Based on surface wave excitation we show in Fig. 7 an excitation of a double meander matrix by a subwavelength source. The sub-wavelength source excites in the first MMS layer resonantly surface plasmons

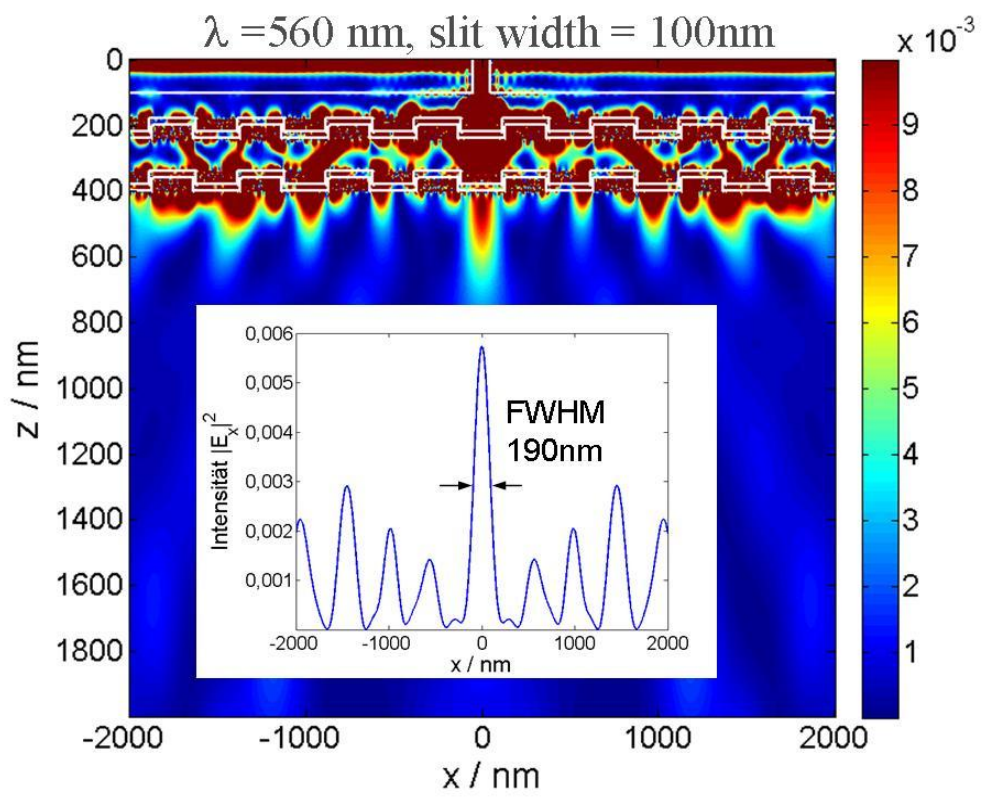

Fig. 7:

Near field profile of a sub-wavelength object after imaging by a double Ag-MMS perfect lens in vacuum [54]. Inset: Intensity profile at $\mathrm{z} \sim \lambda \square$. Structural data (parameter defini-tion see Fig. 3) $\mathrm{P}_{\mathrm{x}}=500 \mathrm{~nm}$; $\mathrm{D}=40 \mathrm{~nm} ; \mathrm{d}(\mathrm{Ag})=20$ $\mathrm{nm} ; \quad \mathrm{D}_{\text {spa }}=100 \mathrm{~nm}$. Material parameters see $[69,70]$.

which are resonantly coupled to the second surface. Despite of the weak oscillation amplitude in the first layer one observes huge plasmon amplitude in the second MMS structure due to resonant coupling. The field distribution of the sub-wavelength source is then imaged in the free space below the $2^{\text {nd }}$ meander surface as already reported for matrices with spherical inclusions in the matrix [76]. The inset shows the sub-wavelength imaging effect. For a working wavelength of $560 \mathrm{~nm}$ we obtain an FWHM of $190 \mathrm{~nm}$ for a slit width of $100 \mathrm{~nm}$. The description of the present material sheet (Au-metallo-dielectric double meander layer) in terms of effective parameters appears challenging. Since due to inversion symmetry in the double meander layers $1^{\text {st }}$ order effects can be assumed to be cancelled by symmetry [39] and resonant quadrupolar contributions due to $2^{\text {nd }}$ order weak spatial dispersion (WSD) effects (Eq. 3a,b) must be taken into account. The quadrupolar contributions can be comparable to dipolar contributions [40] in case of resonance as analyzed for dimers [34]. Therefore a simple 2-parameter description with effective material parameters $(\varepsilon$ and $\mu$ ) can be critical. Over all, at a first glance the double meander structure seems to behave as a NIM slab fulfilling the perfect lens behaviour. This implies also that a double meander structure fulfils the condition of a complementary material which results in a zero optical length between object and imaging (total refractive index appears as zero [53]). On the same footing one can try to combine the PLT with transformation optics to obtain magnification [14, 21, and 22]. An approach can be realized by introducing curved surfaces [10, 71]. Instead curved surfaces, already analyzed in [21-24] another quite general approach is based on zoomed lateral gratings [71]. With respect to the constitutive material equations there seems a difficulty to describe a system due to the absence of inversion symmetry in zoomed gratings, since bi-anisotropic effects [39] can show up destroying negative index behaviour $[33,34]$. On the other hand the resonant plasmon excitation in resonantly coupled MMS can give different weights on the coefficients of $1^{\text {st }}-$ and $2^{\text {nd }}-$ order magnetoelectric coupling. We tested the concept numerically using RCWA [61, 62] discussed also in this conference by Schau [71]. Analyzing an MMS stack with different periods (input period $200 \mathrm{~nm}$, output period $400 \mathrm{~nm}$ ) we demonstrate that enhanced transmission $(80 \%)$ at the passband of the $400 \mathrm{~nm}$ MMS structure (output surface) can be achieved if metal thicknesses and 
corrugation depths of the MMS stack are suitably chosen [54]. The present results on imaging with magnification do not represent the complete solution for a hyperlens on the basis of meander stacks. These first results are however promising as the magnification function seems realizable without relying on a continuous NIM structure [10]. The result seems also robust as realistic material data according to Johnson-Christy [56] were taken into account.

\section{Perfect absorber layers}

The FSS concept mentioned above can be used to suppress back-reflection from dielectric lossy layers. This can be useful for cloaking purposes [26, 27] and stealth technologies [39, chap. II] Especially MTM structures with $\Omega$-shaped inclusions (Omega materials [39]) were of interest in the past. These structures are also of fundamental interest in spectroscopic applications (suppression of stray light) and sensor technology. Very recently new polarization independent designs [77] where coming up. In these designs polarization independent impedance matching was obtained by simple flat Ag cylinders in conjunction with an absorbing thick Ag-layer using the artificial magnetic mode based on the $2^{\text {nd }}$ order WSD-effects (Eq. 3a, b) since the cylindrical array shows inversion symmetry.

\section{What comes next? - A conclusion}

From the present status of the analysis of metamaterials we see that the structure parameter $\mathrm{P} / \lambda$ gives us an important measure to recognize whether pure material effects (artificial permeability and artificial permittivity) or spatial dispersion effects [33, 35] are prevailing in the optical response of nanostructured MTM. e.g. fishnet structures [29, 37]. From the continuous point of view $(P / \lambda \rightarrow 0)$ fascinating possibilities such as perfect lensing [10] (both travelling waves and evanescent waves), super scatters in conjunction with the super phase array [21, 22] to solve, e.g. the compactness-directivity problem of optical antennas can be thought of up to recent proposals in transformation optics as cloaking [24, 26], illusion optics [26, 27, 78] and many more.

We see, however, from the analysis of e.g. wire media [32] that spatial dispersion effects can play an important role and can not be neglected even in the case of very long wavelengths. On the other hand spatial dispersion effects can be used to engineer negative permeability effects [35]. Bianisotropy suitably used can lead to chiral media [39] or Omega media [79] which can be used in applications such as reflectance control [39], perfect absorber [77], or polarization control $[30,80]$.

Apart from the structure symmetry which dictates the spatial dispersion effects we see also that the symmetry of the inclusion in a unit cell influences strongly the optical response via weak dispersion effects (WSD) in dimers [44, 81] or oligmers $[48,50]$ or core shell particles [49]. In MTMs with such inclusions bianisotropic $\left(1^{\text {st }}\right.$ order spatial dispersion effects) might be suppressed with respect to $2^{\text {nd }}$ order dispersion effects realizing pure artificial magnetism.

The selective control of interaction mechanisms in the metamaterials can be especially forced as demonstrated by resonant plasmon coupling effects $[51,53,54]$. An important field in this respect is also resonant plasmon-photon coupling $[16,82-84]$ which paves the route to plasmonic quantum applications.

The strong role of plasmons in metamaterial response reveals another field of application as photonic integration. Since photonic integration possess a natural limit due to the photonic wavelength. Efficient photon-plasmon coupling opens now the path for ultra-dense integrated plasmonic circuits $[20,85,86]$. In analogy to integrated all-photonic circuits [86- 88] all-plasmonic circuits can be thought of more suitable since non-linear effects inherent in the plasmonic system [89] are already present. This would open a new field of Nanoprocessing of optical data comprising analogue techniques (imaging [90]) with digital techniques (integrated plasmonic circuit $[20,86]$ ) with quantum digital techniques) coupling of single photon to single plasmon sources [16, 82] for communication and metrology applications.

\section{Acknowledgements}

The authors would like to thank the Baden-Württemberg Stiftung for financial support.

\section{References and Notes}

[1] W. E. Kock, Metal-Lens Antennas, Proc. of the IRE and Waves and Electrons, 34, 828 (1946)

[2] W.E. Kock, Lens from metal spheres in light-weight matrix, Bell Syst. Technical J. 27, 58 (1948) 
[3] C. Caloz, T. Itoh, Electromagnetic meta materials transmission line theory and microwave applications, Wiley Interscience (2006)

[4] L. Young, L.A. Robinson, C.A. Hacking, Meander-Line Polarizer, IEEE Transaction Antenna Propagat., 376 (1973)

[5] J.F. Zürcher, A Menader-Line Polarizer Covering the Full (E-BAND 60 - 90GHz) MICROWAVE AND OPTICAL TECHNOLOGY LETTERS / Vol. 18, No. 5, 320 (1998).

[6] J.S. Tharp, B.A. Lail, B.A. Munk, and G.D. Boreman, Design and Demonstration of an Infrared Meanderline Phase Retarder, IEEE Trans. Antenna Propagat, VOL. 55, NO. 11, 2983 (2007)

[7] J. Zhou, Th. Koschny, M. Kafesaki, E. N. Economou, J. B. Pendry, and C. M. Soukoulis, Saturation of the Magnetic Response of Split-Ring Resonators at Optical Frequencies, Phys. Rev. Lett. 95, 223902 (2005)

[8] V.G. Veselago, Sov. Phys.-Usp. 10, 509 (1968)

[9] J.B. Pendry, Negative Refraction Makes a Perfect Lens, Phys Rev Lett. 85, 3966 (2000).

[10] J.B. Pendry and S.A. Ramakrishna, Focusing light using negative refraction, J. Phys.: Condens. Matter. 15, 6345 (2003)

[11] H. Raether, Surface Plasmons on Smooth and Rough Surfaces and on Gratings, Springer-Verlag, Berlin (1988).

[12] S. Maslovski, and S. Tretyakov, Phase conjugation and perfect lensing, J. Appl. Phys. 94, 4241 (2003)

[13] S. Maslovski, S. Tretyakov, and P. Alitalo, Near-field enhancement and imaging in double planar polaritonresonant structures, J Appl. Phys. 96, 1293 (2004)

[14] W. H.Wee and J. B. Pendry, Looking beyond the Perfect Lens, Proc. Fourth International Congress on advanced Electromagnetic Materials in Microwaves and Optics, 2010 Metamorphose-VI, p. 623 ISBN 978-952-92 -7734-6623. W.H. Wee and J. B. Pendry, Looking beyond the Perfect Lens, New J. Phys. 12, 053018 (2010)

[15] L.D. Landau, E.M. Lifshitz, Electrodynamics of continuous media $2^{\text {nd }}$ edn. (Oxford Pergamon)

[16] R. Kolesov, B. Grotz, G. Balasubramanian, R.J. Stöhr, A.A.L. Nicolet, P.R. Hemmer, F. Jelezko, J. Wrachtrup, Wave-particle duality of single surface plasmon polaritons, Nature Physics 5, 470 (2009)

[17] W.L. Barnes, A. Dereux, and T.W. Ebbesen, Surface plasmon sub-wavelength optics, Nature 424, 824 (2003)

[18] W. Bogaerts, R. Baets, P. Dumon, V. Wiaux, S. Beckx, D. Taillaert, B. Luyssaert, J. Van Campenhout, P. Bienstman, and D. Van Thourhout, Nanophotonic waveguides in Silicon-on-Insulator fabricated with CMOS technology, J. Lightwave Technol. 23, 401-412 (2005); M. B. Small, D. J. Pearson, On-chip wiring for VLSI: status and directions, IBM Journal of Research and Development, Vol. 34, Issue 6, p. 858 - 867 (1990).

[19] E. Ozbay, Plasmonics: Merging Photonics and Electronics at Nanoscale Dimensions, Science 311, 189 (2006)

[20] S.I. Bozhevolnyi, V.S. Volkov, E. Dvaux, J.Y. Laluet, and T.W. Ebbesen, Channel plasmon subwave length waveguide components including interferometers and ring resonators, Nature 440, 508-511 (2006).

[21] W.H. Wee, J.B. Pendry, Shrinking optical devices, New J Phys. 1, 073033 (2009)

[22] W.H. Wee, J.B Pendry, Super phase array, New J Phys. 12, 033047 (2010)

[23] U. Leonhardt, T.G. Philbin, General relativity in electrical engineering New J Phys. 8, 247 (2006)

[24] U. Leonhardt, T.G. Philbin, Transformation optics and the geometry of light, Prog. Opt. 53, 69 (2009)

Proc. of SPIE Vol. $8083808302-11$ 
[25] T. Yang, H. Chen, X. Luo, H. Ma, Super scatterer: enhancement of scattering with complementary media Opt. Express. 16, 18545 (2008)

[26] Y. Lai, H. Chen, Z.-Q.Zhang, C. Chan, Complementary media invisibility cloack that cloacks objects at a distance outside the cloacking shaell, Phys. Rev. Lett. 102, 093901 (2009)

[27] G.W. Milton, N.-A. Nicrovici, R.C. McPhedran, K. Cherednichenko, Z. Jacob, Solutions in folded geometries and associated cloaking due to anomalous resonance, New J. Phys. 10, 115021 (2008)

[28] S. Hrabar, Waveguide experiments to characterize properties of SNG and DNG metamaterials, chap. 3, p. 87, In Metamaterials Physics and Engineering Explorations, Eds.: N. Engheta, R.W. Ziolkowski, IEEE Wiley Interscience (2006)

[29] N. Liu, H. Guo, L. Fu, S. Kaiser, H. Schweizer, H. Giessen, Three-dimensional photonic metamaterials at optical frequencies, Nature Materials 7, 31 (2008)

[30] N. Liu, L. Fu, S. Kaiser, H. Schweizer, H. Giessen, Plasmonic Building Blocks for Magnetic Molecules in Three -Dimensional Optical Metamaterilas, Adv. Mater. 20, 3859 (2008)

[31] B. Gompf, J. Braun, T. Weiss, H. Giessen, and M. Dressel, Periodic Nanostructures: Spatial dispersion mimics chirality, Phys. Rev. Lett. (2011) (accepted for publication)

[32] P.A. Belov, R. Marques, S.I. Maslovski, I.S. Nefedov, M. Silveirinha, C.R. Simovski, and S.A. Tretyakov, Strong spatial dispersion in wire media in the very large wavelength limit, Phys. Rev. B 67, 113103 (2003)

[33] C.R. Simovsky, On electromagnetic characterization and homogenization of nanostructured metamaterials, J. Opt. 13, 013001 (2011)

[34] C.R. Simovski, S.A. Tretyakov, On effective electromagnetic parameters of artificial nanostructured magnetic materials, Photonics Nanostruct.-Fundamentals and Applications 8, 254 (2010)

[35] C. Menzel, T. Paul, C. Rockstuhl, T. Pertsch, S. Tretyakov, F. Lederer, Validity of effective material parameters for optical fishnet metamaterials, Phys. Rev. B 81, 035320 (2010).

[36] C. Menzel, T. Paul, C. Rockstuhl, R. Lliew, F. Lederer, High symmetry versus optical isotropy of a negative -index metamaterial, Phys. Rev. B 81, 195123 (2010)

[37] J. Valentine, J. Li, T. Zentgraf, G. Bartal, and X. Zhang, Three-dimensional optical metamaterial with a negative refractive index, Nat. Mater. 8, 568 (2009)

[38] C. Altman and K. Suchy, Reciprocity, Spatial Mapping and Time Reversal in Electromagnetics, Developments In Electromagnetic Theory And Applications, Kluwer Acadamic Publishers (1991)

[39] A. Serdyukov, I. Semchenko, S. Tretyakov, and A. Sihvola, Electromagnetics of Bi-anisotropic Materials Theory and Applications, Electocomponent Science Monographs, Gordon and Breach Science Publishers Vol. 11 (2001).

[40] J. Petschulat, C. Menzel, A. Chipouline, C. Rockstuhl, A. Tünnermann, F. Lederer, T. Pertsch, Multipole approach to metamaterials, Phys. Rev. A 78, 043811 (2008)

[41] D.R. Smith, S. Schultz, P. Markos, C.M. Soukoulis, Determination of effective permittivity and permeability of metamaterials from reflection and transmission coefficients, Phys Rev B 65, 195104 (2002)

[42] W. B. Weir, Proc. IEEE 62, 33 (1974)

[43] A. M. Nicholson and G. F. Ross, IEEE Trans. Instrum. Meas. IM-19, 377 (1970)

[44] P. Nordlander, C. Oubre, E. Prodan, K. Li, M.I. Stockman, Nano Lett. 4, 899 (2004)

Proc. of SPIE Vol. $8083808302-12$ 
[45] H. Liu, J.X. Cao, and S.N. Zhu, N.liu, R. Ameling, and H. Giessen, Lagrange model for the chiral optical properties of stereomaterials, Phys. Rev. B 81, 241403 (2010)

[46] According [39] is the splitting of induced currents in electric and magnetic polarization parts not unique up to an arbitrary differentiable vector function $\mathrm{Q}$.

$$
\begin{array}{cccc}
\text { (46.1) } & \vec{P}^{\prime}=\vec{P}+\nabla \times \vec{Q} ; & \text { (46.2) } & \vec{M}^{\prime}=\vec{M}-\partial \vec{Q} / \partial t \\
\text { (46.3) } & \vec{D}^{\prime}=\vec{D}+\nabla \times \vec{Q} ; & \text { (46.4) } & \vec{H}^{\prime}=\vec{H}+\partial \vec{Q} / \partial t
\end{array}
$$

and

$$
\text { (46.5) } \quad \vec{D}=\varepsilon_{0} \cdot \vec{E}+\vec{P} ; \quad \text { (46.6) } \quad \vec{H}=\mu_{0}^{-1} \cdot \vec{B}-\vec{M}
$$

$\mathrm{Q}$ can be used in case of spatial dispersion to transform the constitutive equation to a form where standard boundary conditions for the transformed fields as $\mathrm{E}, \mathrm{H}$ continous transversal components and D, B continuous normal field components. The form of the induced current remains invariant (46.7)

$$
\text { (46.7) } \vec{J}_{\text {ind }}^{\prime}=\partial \vec{P}^{\prime} / \partial t+\nabla \times \vec{M}^{\prime}=\partial \vec{P} / \partial t+\nabla \times \vec{M}=\vec{J}_{\text {ind }}
$$

This fact is also important in metrology and introscopy where detailed information about an electromagnetic medium or MTM-structure shall be obtained.

[47] R. Marque's, F. Medina, and R. Rafii-El-Idrissi, Role of bianisotropy in negative permeability and left-handed metamaterials, Phys. Rev. B 65, 144440 (2002)

[48] A. Alu, A. Salandrino, and N. Engheta, Negative effective permeability and left-handed materials at optical frequencies, Opt. Express 14, 1557 (2006)

[49] C. Rockstuhl, F. Lederer, C. Etrich, T. Pertsch, and T. Scharf, Design of an Artificial Three-Dimensional Composite Metamaterial with Magnetic Resonances in the Visible Range of the Electromagnetic Spectrum, Phys. Rev. Lett. 99, 017401 (2007)

[50] M. Hentschel, M. Saliba, R. Vogelgesang, H. Giessen, A.P. Alivisatos, and N. Liu, Transition from Isolated to Collective Modes in Plasmonic Oligomers, Nanoletters 10, 2721 (2010)

[51] B. Sepulveda, J.B. Gonzalez-Diaz, A.Garcia-Martin, L.M. Lechuga, and G. Armelles, Plasmon-induced Magneto-optical activity in nanosized gold disks, Phys. Rev. Lett. 104, 147401 (2010)

[52] T. Weiss, N. A. Gippius, G. Granet, S. G. Tikhodeev, R. Taubert, L. Fu, H. Schweizer, H. Giessen, Strong resonant mode coupling of Fabry-Perot and grating resonances in stacked two-layer systems, Photonics and Nanostructures - Fundamentals and Applications to be published (2011), doi: 0.1016/j.photonics.2011.03.007

[53] L. Fu, H. Schweizer, T. Weiss, and H. Giessen, Optical properties of metallic meanders, J. Opt. Soc. Am. B 26, B111 (2009).

[54] H. Schweizer, L. Fu, M. Hentschel, T. Weiss, C. Bauer, P. Schau, K. Frenner, W. Osten, and H. Giessen, Resonant Multimeander-Metasurfaces: A Model System for Superlenses and Communication Devices, Phys. Stat. Sol. (b) 2011 (to be published).

[55] T. Okamoto, J. Simonen, and S. Kawata, Plasmonic band gaps of structured metallic thin films evaluated for a surface plasmon laser using the coupled-wave approach, Phys Rev. B 77, 115425 (2008);

I.R. Hooper, J.R. Sambles, Coupled surface plasmon polaritons on thin metal slabs corrugated on both surfaces, Phys. Rev. B 70, 045421 (2004).

[56] P. B. Johnson and R. W. Christy, “Optical constants of the noble metals," Phys. Rev. B 6, 4370-4379 (1972).

[57] P. Andrew and W. L. Barnes, Energy Transfer Across a Metal Film Mediated by Surface Plasmon Polaritons, Science 306, 1002 (2004). 
[58] M. Yoshida, S. Tomita, H. Yanagi, S. Hayaski, Resonant photon transport through metal- insulator-metal multilayers consisting of Ag and SiO2, Phys Rev B 82, 045410 (2010).

[59] L. Fu, H. Schweizer, H. Guo, N. Liu, and H. Giessen, Appl. Phys. B 86, 425 (2007).

[60] H. Schweizer, L. Fu, H. Gräbeldinger, H. Guo, N. Liu, S. Kaiser, and H. Giessen, phys stat. sol. (b) 244, 1243 (2007).

[61] T. Weiss, N. A. Gippius, S. G. Tikhodeev, G. Granet, and H. Giessen, Efficient Calculation of the Optical Properties of Stacked Metamaterials with a Fourier Modal Method, J. Opt. A 11, 114019 (2009).

[62] P. Götz, T. Schuster, K. Frenner, S. Rafler, and W. Osten, Normal vector method for the RCWA with automated vector field generation, Opt. Express 16, 17295-17301 (2008).

[63] T. Schuster, J. Ruoff, N. Kerwien, S. Rafler, and W. Osten, ,Normal vector method for convergence improvement using the RCWA for crossed gratings, J. Opt. Soc. Am. A 24, 2880 (2007).

[64] E. Popov, M. Neviere, 'Maxwell equations in Fourier space: fast-converging formulation for diffraction by arbitrary shaped, periodic, anisotropic media,' J. Opt. Soc. Am. A 18, 2886 (2001).

[65] L. Li, 'New formulation of the Fourier modal method for crossed surface-relief gratings', J. Opt. Soc. Am. A 14, 2758 (1997).

[66] G. Granet, 'Reformulation of the lamellar grating problem through the concept of adaptive spatial resolution', J. Opt. Soc. Am. A 16, 2510 (1999).

[67] G. Granet, J.P. Plumey, 'Parametric formulation of the Fourier modal method for crossed surface-relief gratings', J. Opt. A: Pure Appl. Opt. 4, 145 (2002).

[68] A. Aubry, J.B. Pendry, Mimicking a negative refractive slab by combining two phase conjugators, J. Opt. Soc. Am. B Vol. 27 (1), 72 (2010).

[69] In our simulation we used a Drude model with fitted parameters $\left(\omega_{\mathrm{p}}=1.371016 \mathrm{rad} / \mathrm{s}, \gamma=0.851014 \mathrm{rad} / \mathrm{s}\right)$ for $\mathrm{Ag}$ from [52] to keep our data comparable with other published data. The comparison of experimental with theoretical Au-MMS structures has been carried out with Johnson-Christy dispersion data [53].

[70] G. Dolling, C. Enkrich, M. Wegener, C. M. Soukoulis, and S. Linden, "Low-loss negative-index metamaterial at telecommunication wavelengths," Opt. Lett. 31, 1800-1802 (2006).

[71] P. Schau, K.Frenner, L. Fu, H. Schweizer, W. Osten, doi:10.111/12.854214, pp.77111F-77111F-10 (2010).

[72] Z. Liu, H. Lee, Y. Xiong, C. Sun, X. Zhang, Far-Field Optical Hyperlens Magnifying Sub-Diffraction-Limited Objects, Science 315, 1686 (2007).

[73] Z. Jacob, L.V. Alekseyev, and E. Narimanov, Optical Hyperlens: Far-field Imaging beyond the diffraction limit, Opt. Express 14, 8247 (2006).

[74] L.V. Alekseyev and E. Narimanov, Slow light and 3D imaging with non-magnetic negative index systems, Opt. Express 14, 11184 (2006).

[75] O. Malyuskin, and V. Fusco, Far Field Sub-wavelength Source Resolution Using Phase Conjugating Lens Assisted With Evanescent-to-Propagating Spectrum Conversion T-AP 58, 459 (2010).

[76] C.R. Simovski, A.J. Viitanen, S.A. Tretyakov, Sub-wavelength resolution in linear arrays of plasmonic particles, J. Appl. Phys. 101, 123102 (2007).

[77] N. Liu, M. Mesch, T. Weiss, M. Hentschel, and H. Giesen, Infrared Perfect Absorber and ist Application as Plasmonic Sensor, Nano Lett. 10, 2342 (2010) 
[78] J. B. Pendry, D. Schurig, D. R. Smith, Controlling Electromagnetic Fields, Science 312, 1780 (2006)

[79] S.A. Tretyakov, C.R. Simovski, M. Hudlička, Bianisotropic route to the realization and matching of backwardwave metamaterial slabs, Phys. Rev. B 75, 153104 (2007)

[80] E. Plum, X.-X. Liu, V.A. Fedotov, Y. Chen,, D.P. Tsai, and N.I. Zheludev, Metamaterials: Optical Activity without Chirality, Phys. Rev. Lett. 102, 113902 (2009)

[81] H. Liu, J.X. Cao, and S.N. Zhu, N. Liu, R. Ameling, and H. Giessen, Lagrange model for the chiral optical properties of stereo metamaterials, Phys. Rev. B 81, 241403 (2010)

[82] A.V. Akimov, A. Mukherjee, C.L. Yu, D.E. Chang, A.S. Zibrov, P.R. Hemmer, H. Park, M.D.Lukin, Generation of single optical plasmons in metallic nanowires coupled to quantum dots, Nature 450, 402 (2007)

[83] Y. Fedutik, V.V. Temnov, O. Schöps, U. Woggon, Exciton-Plasmon-Photon Conversion in Plasmonic Nanostructures, Phys. Rev. Lett. 99, 136802 (2007)

[84] D. E. Chang, A. S. Sørensen, P. R. Hemmer, and M. D. Lukin, Quantum Optics with Surface Plasmons, Phys Rev. Lett. 97, 053002 (2006).

[85] A.V. Zayats, and I. I. Smolyaninov, Near-field photonics: surface plasmon polaritons and localized surface plasmons, J. Opt. A: Pure and Appl. Opt., 5, S16-S50 (2003).

[86] J. Dionne, L. Sweatlock, and H. Atwater, "Plasmon slot waveguides: Towards chip-scale propagation with subwavelength-scale localization,” Phys. Rev. B 73, 035407 (2006).

[87] A.E. Willner, B. Zhang, L. Zhang, L. Yan, I. Fazal, Optical Signal Processing Using Tunable Delay Elements Based on Slow Light," IEEE J. Sel. Top. Quant. Electron. 14, 691 (2008).

[88] J. B. Khurgin, Optical buffers based on slow light in electromagnetically induced transparent media and coupled resonator structures: comparative analysis, J. Opt. Soc. Am. B 22, 1062 (2005).

[89] G. A. Wurtz, R. Pollard, and A.V. Zayats, Optical Bistability in Nonlinear Surface-Plasmon Polaritonic Crystals Phys. Rev. Lett. 97, 057402 (2006);

K. Ujihara, PhaseE Conjugation of Surface Plasmon Waves by the Third Order Nonlinearity of a Free Electron Gas, Opt. Commun. 43, 225 (1982);

A. Marini, A. V. Gorbach, D. V. Skryabin, and A. V. Zayats, Amplification of surface plasmon polaritons in the presence of nonlinearity and spectral signatures of threshold crossover, Opt. Lett. 34, 2864 (2009)

[90] I.I. Smolyaninov, J. Elliott, A.V. Zayats, and C.C. Davis, Far-field optical microscopy with a nanometer-scale resolution based on the in-plane image magnification by surface plasmon polaritons, Phys Rev Lett. 94, $057401(2005)$. 